\title{
Economy at Cardiologic Risk
}

\author{
Roshan Prasad Adhikari ${ }^{1}$ \\ ${ }^{1}$ PhD Scholar, Dr. K.N. Modi University, Newai, Rajasthan, India
}

\begin{abstract}
Cardiovascular disease should be prevented or overcome early period in order for letting expectable role in welfare package and rich contribution within the unitary construct of sociocultural system when structure and function are reciprocated to by the inherent activity of an individual member. This research work is mixed, exploratory, with description of primary and secondary data rather a qualitative. Interrelationships among cardiovascular system, social and cultural factors are assessed and measured with standard at personal, familial and social background as known that incapacitation out of heart attack onslaught on eligibly participating candidates can threaten conducive affinity cast on track of progress; the attained disability does not allow which. An accumulation of total 330 respondents of four areas include coronary artery disorder of illiterate, literate, well educated, adopt and intellectual denizens. Their collection of altogether 330 coronary heart patients in four areas had included both arrogant and studious type of people. Among the victims 30 were pilot and 300 subject people for post study. Their $78.3 \%$ were literate, $64.8 \%$ intentional about socio-cultural performances where $25 \%$ had involved and whose participation 58.3\% locals ever requested since remembered the previous many contributions at $43.9 \%$ degree. Their performances on various societal fields and prospective benefit which the heart complication if onslaught would finish up to where human expertise were mainly useful and an again local demands of those have indicated probability of surrounding gains during inclusion but development lag should have taken place instead of. Present researcher has faith on an actual transformation into systemic modification where societal process can meet culmination point, more than mental and physical health must remain sound, social terms coped better, and culture cherished through a consistent mutuality.
\end{abstract}

KEYWORDS: Cardiology, Chronic Arterial, Myocardium

\section{INTRODUCTION}

This study focuses on the impact of financial management on maintenance of cardiac fitness along-with socio-cultural accomplishments shown the disease sufferers under medical sociological perspective analysis. It can become possible to analyze the result of coronary disease whatever it gives normally on the personal, familial and socio-cultural set-up. In order to intimate the inter-connection among health, ability, environment and economic condition of any cardiac patient in terms of disability onset remained a trend before single alternative solution that could tackle the social and cultural uplift relating problems to come up from coronary event in mostly qualified because brain toil is the sole risk of this.

Medical treatments against several ailments related to circulatory, nervous, skeletal and respiratory troubles have proved to be good for longevity but preventive and not curative measures adopted by implantation, surgical and transplantation therapies can help liven the patient for a certain period with chronic arterial defects out of several examples over past and present. In order to extirpate heart disease by diabetic root an immunization drug otelixizumab was invented recently in one of the developed countries that will be processed after its five years. Diabetes is directly associated with a marked increase of myocardial infarction that results from 
the rupture of tissue of heart muscle called myocardium when blood will not reach there sufficiently. Patients with diabetes not having myocardial infarction are always treated for cardiovascular causes as those having it. Far better way to assess whether patients with sugar and ones clinically subjected to coronary heart disease can have the same risk of cardiovascular sufferings is through comparing the risk of such occurrences in diabetic symptoms with and without prior coronary problem with that in non-diabetic cases with and without prior records. Patients with diabetes mellitus have a good basis of treatment against cardiovascular risk factors aggressively safe from cardiac arrest by rupture of myocardium the muscle of heart. In previous reports, the excess risk of coronary diseases in people with prior myocardial infarction calling cardiac arrest was higher than the excess risk in diabetic ones. Lifestyle changes, in purpose, have been shown to be effective in reducing, and in the case of diet also reversing, coronary artery disease with a control over sugar and blood pressure levels. Healthy diet has been shown along with adoption of other life-style changes oriented to be hugely effective as a treatment of coronary disease, and generalized atherosclerosis due to arterial obstruction of blood after plaque formation. Life style should have fixed weight control, smoking cessation, bodily exercise, fish oil consumption to increase omega-3 fatty acid intake, food products derived from cow milk and avoidance of sedentary habit for a vulnerable patient.

However, signs of complete thrombosis such as diffuse swelling of the extremity may appear late and their absence does not exclude the diagnosis of the abundance of excess liquid content in blood vessels. Complex congenital heart disease is defined as cyanotic or a cyanotic structural congenital heart disease other than a simple atrial, septal defect, a ventricular septal defect, or a patent ductus arteriosus. The clinical presentation of candidemia included fever in all of the patients. Fever persisted at least intermittently, despite appropriate antifungal therapy. Five of the diseased presented with thrombocytopenia at the onset of candidemia. All patients developed swelling of the extremities related to thrombosis of a great vein at which point further investigations were performed to evaluate for fluid retention. Edema corresponding to the involved vein has been described in cases of candida septic thrombosis of the great veins in adults (Chandrama Chakrabarti, 2012, pp. 753-757). Thus, a combination of factors including persistent fever during or after the use of broad-spectrum antibiotics against the chronic infection despite antifungal therapy, and prolonged edema of the affected limb, suggests candida thrombophlebitis of a central vein together-with documentation of the thrombus because avoids mortality.

The circulatory system inside human body, when hindered by plaques composed of substances namely fat, lipid, sugar, pressure hormones, amino and uric acid salts, etc., into cardiac organ blood carrying such nutrients cannot reach to. According to clinic arterial blockage, heart defects will develop symptoms of angina pectoris or chest pain, atherosclerosis, ventricular arrhythmia, transient ischemia, and myocardial infarction (Clinic, 2010). This stage destines in victim's irreversible death unless organ, stent, and pace maker any transplantation otherwise stenography and angiotherapy will be lately resorted to. Early precautions, hence, are most essential that lead all to live freely from coronary complaints and allied with negative impacts on health, body work and collectivities of other people. Purposefully a suitable prevention before cure possibility against heart disease under various discoveries from allopathic, homeopathy, naturopathy, physiotherapy, alternative therapy using several healing methods may open blood flow to heart slowly compared to instant benefits had from coronary interventions and bypass surgery. 
A social worker aloof from diseased cardiac and cerebral organs can ever remain capable into co-operating with benevolent actions for familial, social, and cultural pleasures up-to the same one's inherited capacity unless gone excluded because of caste, creed, ethnic, tribal and communal identifications. The fundamental element of socialization is peoples' communication which is produced by web of interactive roles within an organized mass of people. The individual is relevant to social affairs who should intervene in communicative behavior can be interpreted as participating in a relationship among other such citizens. If the secret aspects of any individual are hindered to get communicated, or not understood in a communicative fashion by others, will not appear social, instead part of the environment which may disturb the whole system of social behaving. The more the quantity together with quality of manpower resources as well as economic infrastructure the sounder the mechanism of social structural functional relationship of life setting can get manifested: through sustenance caused of sole combined activities.

\section{METHODS}

Where things ruin psychologically in the course of social research, the undertaken task must care for the subtlest dangers and guard against them asking to reveal deviant nature, attitude feelings are unpopular, personal characteristics that may seem demeaning, such as low income, the receipt of welfare payments, etc. Detailing ideas usually make subjects happen at least uncomfortable and social research should never injure target people, regardless of whether they volunteer in purpose of. Perhaps the clearest instance of this norm practically concerns the information that would embarrass subjects or jeopardize their privacy, friendships, jobs, so forth. Social research projects may force participants to face their aspects that are not normally considered. This can happen again when the information is unrevealed directly to the researcher. The project then can continue arousing individual's sentiment behind the same curiosity to learn which cherished ever before sole inquiry of a targeted score. If the study is concerned on codes of ethical conduct, for example, the text may begin questioning anybody's own mortality, and that personal concern may last long having later on the research completed.

\section{RESULTS}

\section{Economic State}

Table 1: Economic State Economic State

\begin{tabular}{|l|l|l|l|l|l|}
\hline \multicolumn{2}{|c|}{} & Frequency & Percent & Valid Percent & $\begin{array}{l}\text { Cumulative } \\
\text { Percent }\end{array}$ \\
\hline \multirow{3}{*}{ Valid } & Sustainable & 170 & 56.7 & 56.7 & 56.7 \\
\cline { 2 - 6 } & Unsustainable & 114 & 38.0 & 38.0 & 94.7 \\
\cline { 2 - 6 } & Don't know & 16 & 5.3 & 5.3 & 100.0 \\
\hline Total & 300 & 100.0 & 100.0 & \\
\hline
\end{tabular}

Sources: Field Survey, 2013

The frequency of respondents whose economic state sustains their livelihood is 170 at $56.7 \%$ with 59.9 the cumulative percentage, that of whose economic state does not sustain this is 114 at $38.0 \%$ with 100.0 the cumulative percentage, since the same values are proved as significant. Similarly the total system of respondents has a frequency of 284 at $94.7 \%$, missing ones has it of 16 at $5.3 \%$ and their total frequency becomes 300 at $100.0 \%$.

2. Self-funding 
ISSN: 2362-1303 (Paper) | eISSN: 2362-1311(Online)

JOURNAL OF ADVANCED ACADEMIC RESEARCH (JAAR)

July 2014

Table 2: Self-funding

\begin{tabular}{|c|c|c|c|c|c|}
\hline \multicolumn{6}{|c|}{ Self-funding } \\
\hline & & Frequency & Percent & Valid Percent & Cumulative Percent \\
\hline \multirow{4}{*}{ Valid } & yes & 85 & 28.3 & 28.3 & 28.3 \\
\hline & No & 182 & 60.7 & 60.7 & 89.0 \\
\hline & \begin{tabular}{|l} 
Do \\
Know
\end{tabular} & 33 & 11.0 & 11.0 & 100.0 \\
\hline & Total & 300 & 100.0 & 100.0 & \\
\hline
\end{tabular}

Sources: Field Survey, 2013

The respondents who if were unsupported from any side could follow the treatment by their own economy made a frequency 85 at $28.3 \%$ with 28.3 the cumulative percentage, who could not follow had a frequency 182 at $60.7 \%$ with 89.0 the cumulative percentage, and those who are unknowing of the answer have a frequency 33 at $11.0 \%$ with 100.0 the cumulative percentage which had given a valid result. Their total frequency has become 300 at $100.0 \%$.

It is obvious from the literature that a disabled state because of traumatized heart organ gives rise to abnormal behavior of the sufferer person that can be treated by medical therapies. Abnormality is attributive to handicapped state of physical organs later and the same health condition because of acquired debility will produce psychosomatic alterations in one's personal manners. If the behavior is sociable it will turn into a modal personality of the candidate but remaining influenced by socio-economic conditions which show reliance on income level after physical impairment from heart troubles. There will thus remain a chain of events since the poverty causes disease and vice versa if expenditure is not enough affording for preventive care of health otherwise heart related debility starts on a qualified who had previously been involved in certain earning businesses.

\section{DISCUSSION}

Instead of the better situation by homes serious heart patients survive burdensomely to their family when wheel chair bound, bed ridden or confined inside a house never physically capable enough of earning, indulging in social and cultural affairs, performing innovative tasks conducive to the national development even if they are traditionally and academically rich of essential talents. Many aspects of human development draw the working mechanism toward a crucial path active since because of creating double way complexity within a contour of various types of goal. Material, economic and work relations help affect the philosophical reflection in a socio-cultural arena until construct varied norms and values of preceding a new organization. Gaarder describes three levels in bases of society are the most fundamental aspects called its conditions of production. In other words the natural conditions or resources that are available to society are foundation of any society and this clearly determines the type of production therewith.

The daily life activities of humans are thought to be terminated in a condition of financial management remaining according to one's income level. The sustenance of individual and household expenses should be defined in terms of capacity to earn money by virtue of qualification, eligible health and physical fitness of a person. When a person is rich in talent, physique, skill and literacy but lacks the good position of health the action useful to boost up environmental construct may not be expectable from such a being. The speed of social change toward a desired destination is happened out of unanimous steps of potent workers of a holistic 
system in a sense of again possessing a good health, education and bodily organs. All of the required aspects to take part in role making along human uplift are backed by vehement source of fund because qualities of individual life are in other terms liable to be earned at labor as well as an exchange of money with some item. In different words it is said an individual should maintain own disease free state, study level, physical set-up with an expenditure from one's very child life instead it may pose a big threat on personal uplift any day of outreach made. It is actually become possible doing in different fashions such as of self-income source, household support institutional favor and government approval for those who associate with social and political systems within a country. The ones devoid of all these sides do not happen to afford for the basic need satisfaction and together-with educational achievement as well as life expectancy health continuity remains at a challenged turn for being like unsupported status. Financial condition for a person proves as if a pillar for holding the consistency of daily life management had in care about food, cloth shelter prior to run business activities. A person acquires statuses related with job, study, societal affairs but cannot happen to indulge into responsibilities unless becoming backed by fund resource, similarly then hankers after income source when someone will turn able enough. In this example the main role of economic system has seemed as a strong pillar to balance the achievements supposing from various sectors of development for human aggregate. The endeavors just paid have been united about well-designed plan are oriented to the welfare of an individual, family and society or community under an availability of expenditure amount that is the target success which is feasible but its definite course there completes together-with. Desired achievement relevant to specific field of excellence in need of holistic demand satisfaction that pertains to positive side of aim for change process paves a road to a nearly period by fund coped with similarity. The overall increase in speed of progress planned under systematic investigation of surrounding environment of human habitat takes place reliant on utilization of power as well as material resources of natural and social worlds. The natural treasure is able to avail soil, water, plant, electricity, sunlight, food produce, etc. while social and cultural accomplishment affords to ease a human with material as well as immaterial possessions. The life of a community of people is better facilitated in case the factors of construction building such as man power and infrastructure resources are met by society concerned needs and conditions for developmental scheme because the existence of human beings in an environmental system is expected from the level of production in several fields. Investment in health is not only a desirable, but also an essential priority for most societies. However, our health systems face tough and complex challenges, in part derived from new pressures, such as ageing populations, growing prevalence of chronic illnesses, and intensive use of expensive yet vital health technologies. The effects of health on development are clear. Countries with weak health and education conditions find it harder to achieve sustained growth. Indeed, economic evidence confirms that a $10 \%$ improvement in life expectancy at birth is associated with a rise in economic growth of some 0.3-0.4 percentage points a year (Frenk, 2004).

The socio-economic gradient in coronary heart disease persists across virtually the entire socioeconomic spectrum, with additional reductions in risk observed at even the highest levels of socio-economic position. However less is known about how the socio-economic gradient in incident coronary heart disease may differ between men and women. The socio-economic gradient in health is traditionally believed to be weaker for women than for men, attributed in part to issues of measurement of socio-economic position among women. However, gender differences in associations between socio-economic position and health depend upon a variety of 
factors, including age of participants, marital status, choice of socio-economic indicator, including whether a woman is classified by personal, partner, or household socio-economic position, inclusion of women outside the paid workforce and health outcomes examined. While outcomes such as total mortality exhibits weaker or comparable socio-economic gradients for women relative to men, several studies suggest that socio-economic gradients in coronary heart disease may be stronger in women.

It is an inevitable expression that people abiding by strong ties of familial, cultural and community feeling are able to procure better hygienic possession than those socially isolated since the fact social cohesion or 'connectedness' is related to the health of individuals and communities.

Cardiovascular disease (CVD) is absent from the global development agenda. This absence is striking, because CVD is a major impediment to human development, imposing large health and economic burdens in low- and middle-income countries. These burdens have a reciprocal relationship in that poverty is a potent cause of CVD, whereas CVD contributes to poverty. The present editorial explores and seeks to explain the global neglect of CVD in pursuit of a strategy for its prevention and control worldwide. CVD is also responsible for much disability, often for decades of a person's life. Nearly half of the global burden of disease is caused by chronic diseases, and CVD is the leading contributor among these (al, 2014).

In both the developing and the developed world, men wield enormous power over many aspects of women's lives. In the public sphere, as heads of states and government ministers, as leaders of religious institutions, as judges, and village heads, men design and implement policies that may or may not favor women's priorities and needs. In the private sphere, as husbands and fathers, men can directly impact the economic and social progress of women. Thus, ignoring men in the design and implementation of gender-oriented policies may not only limit the effectiveness of these initiatives but also exacerbate existing disparities (Farre, 2013).

A global CVD epidemic is rapidly evolving, with the burden of disease shifting. Twice as many deaths due to CVD now occur in developing countries as in developed countries. The vast majority of CVD can be attributed to conventional risk factors. Even in sub-Saharan Africa, high blood pressure, high cholesterol, tobacco and alcohol use, and low vegetable and fruit consumption are already among the top risk factors for disease. Because of the lag time associated with CVD risk factors, especially in children, the full effect of exposure to these factors will only be seen in the future. Information from more than 100 countries shows that more 13- to 15-year-olds smoke than ever before, and studies show that obesity levels in children are increasing markedly in countries as diverse as Brazil, China, India, and almost all island states.

Population-wide efforts now to reduce risk factors through multiple economic and educational policies and programs will reap savings later in medical and other direct costs, as well as indirectly in improved quality of life and economic productivity. Until that time, many currently available treatments for acute and chronic management of CVD exist and ought to be considered in developing countries where their use is currently limited (Gaziamo, 2005).

CVD risks arise on the path to an advanced stage of economic development, driven in part by a range of development-related factors such as population aging and urbanization; shifts in agriculture; and the multifarious influences of globalization. CVD can affect the entire economy of a developing nation. Disability, early mortality, and direct health expenditures can divert resources from savings, investment, and other productive uses, affecting economic wellbeing at the household level and growth potential at the national level. Therefore, developing 
countries need to maneuver diligently and carefully to avoid cementing in place long-term roadblocks to healthy economic progress.

The pattern of rising Cardio Vascular Disease (CVD) risks with development can reflect a complex interaction among average per capita income in a country, trends in lifestyle and other risk factors, and health systems capacity to control CVD. Developing countries at different stages of development are found to be facing different challenges in choosing public health strategies to reduce the burden of CVD. The challenge facing low income developing countries is to continue to bring down prevalence of infectious diseases while avoiding an overwhelming rise in CVD, especially under conditions of resource limitations. This will require balancing competing population-level health demands while maintaining relatively low overall health expenditures. Investments in health will also need to be facing challenge and middle income developing countries are to reverse or slow the rise in CVD in an affordable and cost-effective manner.

Social and economic development policies, as Chalk cites, can weaken local support for terrorist activities. Social and economic development policies can contribute to the expansion of a new middle class in communities that have traditionally lent support to terrorist groups. In many cases, this section of the population has recognized the economic benefits of peace and, as a result, has worked to inhibit local support for terrorist activities. Social and economic development can discourage terrorist recruits.

\section{CONCLUSION}

The author accepts the fact that social and economic development policies can help to reduce the pools of potential recruits by reducing their perceived grievances and providing the members of these communities with viable alternatives to terrorism (Chalk, 2005). In order to have a check and balance system in society, economic policy needs to be strengthened as a result of the harmonized activities within the community of people bounded in adapted territory of a nation the proposed system will be modified to a goal.

As per a compromise with the importance of role of finance in social and cultural uplift after all evidences observed the present researcher has equated the following relation freely,

Where,

$$
\mathrm{E}+\mathrm{O} \text { channel }
$$

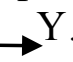

$\mathrm{E}=$ Economy,

$\mathrm{O}=$ Outreach,

Y= Yield.

Meaning: Outreach activities of a person, family members, society and nation if enhanced by economy as a fuel can fetch a yield product depending on the trend of initial speed. Therefore a target plays a vital role in attaining the score of the nature pertaining to collective interest of surroundings even though rest factors will work better.

\section{BIBLIOGRAPHY}

Al, R. B. (2014). The Global Implications of Cardiovascular Disease. Circulation, 1.

Chalk, K. C. (2005). The Role of Social and Economic Development, Global Priorities. RAND, 1.

Chandrama Chakrabarti, S. k. (2012). Prolonged Candidemia In Infants Following Surgery For Congenital Heart Disease. Infection Control and Hospital Epidemiology Vol. 24 No. 10 , 753-757. 
Farre, L. (2013). The Role of Men in Development . Let's Talk Development, 1.

Frenk, J. (2004). Mexican Minister of Health and Chair of the OECD Health Ministers. Sections, Society General , 1.

Gaziamo, T. A. (2005). Cardiovascular Disease in the Developing World and its Cost-Effective Management. Circulation Heart Diseae in Africa, 1.

Staff, M. C. (2010). Life style and home remedies. Mayo Foundation for Medical Education and Research , 1, 2.

Steven M. Haffner, S. L. (1998). Mortality From Coronary Heart Disease In Subjects With Type 2 Diabetes And In Nondiabetic Subjects With And Without Prior Myocardial INfarction. The New England Journal of Medicine , 229,230. 